\title{
Controlled interaction: Strategies for using virtual reality to study perception
}

\author{
Frank H. DURGIN AND ZHI Li \\ Swarthmore College, Swarthmore, Pennsylvania
}

\begin{abstract}
Immersive virtual reality systems employing head-mounted displays offer great promise for the investigation of perception and action, but there are well-documented limitations to most virtual reality systems. In the present article, we suggest strategies for studying perception/action interactions that try to depend on both scale-invariant metrics (such as power function exponents) and careful consideration of the requirements of the interactions under investigation. New data concerning the effect of pincushion distortion on the perception of surface orientation are presented, as well as data documenting the perception of dynamic distortions associated with head movements with uncorrected optics. A review of several successful uses of virtual reality to study the interaction of perception and action emphasizes scale-free analysis strategies that can achieve theoretical goals while minimizing assumptions about the accuracy of virtual simulations.
\end{abstract}

There are two main reasons why the idea of virtual reality (VR) has so deeply appealed to students of perception. The first is the promise of being able to present any largescale environment that is desired. To study basic space perception, for example, researchers can change the horizon line while leaving all other scene geometry correct (Messing \& Durgin, 2005), and they can make large-scale hills of any surface orientation (Creem-Regehr, Gooch, Sahm, \& Thompson, 2004; Li \& Durgin, 2009; Proffitt, Bhalla, Gossweiler, \& Midgett, 1995). The second reason why VR has such appeal is because it simulates, and thus allows us to study, the interaction between behavior and perception (see, e.g., Warren, Kay, Zosh, Duchon, \& Sahuc, 2001). Such interactivity is fundamental, and the control it allows is revolutionary (Loomis, Blascovich, \& Beall, 1999).

Powers (1973) famously proposed that behavior is best construed as the "control of perception." By this, he meant to argue that perceptual feedback is more than a means of guiding action. It is often the proximal goal of action. At the very least, action involves perceptual prediction (Wolpert \& Flanagan, 2001). Immersive virtual environments are intended to capture this interactivity: I turn my head to bring an object of interest into view, and it comes into view. I walk forward to experience a new region of visual space, and there I am. VR systems can be used to alter the perceptual gain of actions, such as walking or rotating, thus respecifying the quantitative relationship between perception and action (see, e.g., Mohler et al., 2007).

With regard to creating any environment desired, VR systems have proven to be disappointing in practice because of their apparent failure to generate the perceptions we expect. Interactivity provides a visceral sense of immersion in a virtual environment that can be quite compelling, even when the computer graphics are clearly artificial. However, the virtual spaces created nearly always appear much smaller than they are simulated to be. This phenomenon is well documented, but is still not well understood (Loomis \& Knapp, 2003; Thompson et al., 2004). If one is studying qualitative spatial cognition, these quantitative errors of scale may not be critical. For example, to study spatial reorientation in a small virtual room, we simply simulated a much larger room in order to achieve the sense of spatial scale that we intended (Grodner, Pietsch, \& Durgin, 2009). But this is not ideal. There is some evidence that interacting with a virtual environment that exactly matches the real environment in which one has just been immersed helps to scale the virtual space (Akagi \& Durgin, 2007; Interrante, Anderson, \& Ries, 2006; Interrante, Ries, Lindquist, \& Anderson, 2007). However, requiring prior exposure to a real version of the virtual space seems to undermine one of the chief virtues of VR: the ability to present any environment desired.

Because the perceived scale of virtual environments can generally be assumed to be different from (smaller than) the simulated scale, studies of space perception in VR are faced with a conundrum: It is possible to manipulate the environmental information to try to investigate the effectiveness of certain kinds of visual information, but the absolute scale is known to be wrong. What counts as accurate, and what counts as inaccurate? It is worth considering whether the second-order scaling of VR (compression of scale with distance) is more or less uniform. If it is, then we can ask questions about the rate of compression such as those that have been traditionally asked in studies of distance perception using scale-free methods (see, e.g., Teghtsoonian \& Teghtsoonian, 1970). The scale-free method of using the exponents of power

F. H. Durgin, fdurgin1@swarthmore.edu 
functions to represent perceptual experience has proven to be very useful in a variety of studies using VR in our lab. In the present article, we will review examples of how we have previously applied the scale-free method in VR to distance perception, self-motion perception, and geographical slant perception. Finally, we will present a novel experiment using the method to demonstrate the perceptual importance of a subtle optical correction in virtual environments.

The perceived distance to an object along the ground may be calculated in terms of its visual declination below the horizontal (Ooi, Wu, \& He, 2001). That is, assuming a level ground plane and a standing observer, there is a direct relationship between how far away an object is from the observer and the visual angle below horizontal where its base appears to meet the ground. Messing and Durgin (2005) sought to test whether subtly lowering a visible horizon (by $1.5^{\circ}$ ) would affect distance estimates in near space (1-7 m). Knowing that distance would be underestimated in virtual reality, and that the absolute shifts in perceived distance would be difficult to detect because of intersubject variability, Messing and Durgin expressed their predictions of horizon effects in terms of differences in the exponents of power functions that were fit to distance estimates with a true (normal) horizon and an artificially low horizon. Although a power function merely approximates the expected perceptual change, lowering the horizon line by $1.5^{\circ}$ predicts an exponent of about 1.15 for this range of distances. Thus, although each participant made 10 distance estimates with each horizon line, the statistics used for analysis simply examined the individual exponents of power functions for each horizon condition by participant. Both for verbal estimates and for action measures (walking to the previewed target location without visual feedback), it was found that although distances were underestimated in all conditions, exponents were normal (near 1) with the correct horizon. This finding suggests that the compression of perceptual space in VR was uniform. Moreover, the shifted horizon reliably produced the predicted change in exponent for both measures. This illustrates the advantage of looking for higher order (scale-free) predictions when studying perception in virtual environments - even when a scaled quantity (i.e., perceived distance) is under investigation.

The ability to create an interaction between action and perception is not unique to immersive virtual reality systems, but the power and generality of the technology makes the study of that interaction much more versatile. Consider that Wallach and Flaherty (1975) built a mechanical device that allowed them to present an expanding flow field (based on a rotating spiral image) that was yoked to the mechanical motion of an observer on a swing, thus allowing them to demonstrate that motion aftereffects were reduced when the motion stimulus (the expanding flow field) was consistent with the forward physical motion of an observer. (No such reduction was found if the expanding flow field was presented on the back swing instead.) This important finding was later replicated using a computer-controlled motion stimulus that was yoked electronically to the motion of a cart carrying an observer (Harris, Morgan, \& Still, 1981). With the advent of immersive VR, such contingencies have become the bread and butter of a variety of studies. For example, using immersive VR, we have quantified the reduction in perceived visual speed during self-motion and have shown that it can be modeled as a subtractive function that is proportional to the speed of self-motion under a variety of conditions (Durgin, Gigone, \& Scott, 2005).

Once again, our initial strategy was to use scale-free representations (exponents of power functions fit to verbal speed estimates) to create a basis for comparing two conditions (static vs. moving observers) rather than trying to directly measure a change in any one particular visual speed. The versatility of immersive virtual reality meant that we could easily compare the amount of speed reduction caused by biomechanical activity (walking on a treadmill) with that from physical self-motion (being carried on a cart) and from the two in combination (walking on solid ground in a wide-area immersive virtual environment). The comparison could be made using the same visual information (virtual environment) and measurement strategies across all of these conditions. The amount of speed reduction in the case of normal locomotion was roughly the sum of the amounts found separately for the biomechanical activity of walking and for (passive) physical translation. Although the scaling of perceived speed based on VR might be distorted in much the same way that visual space is distorted in VR, the theoretical inferences of these studies are not limited by this fact because they focus on second-order (scale-free) relationships.

Similarly, evidence that the perceptual discrimination function for visual speed is altered during self-motion, so that speed sensitivity is tuned to expected visual speeds (Durgin \& Gigone, 2007), is not undermined by the knowledge that the spatial scaling of VR is known to be problematic. Discrimination is discrimination. Similarly, that object motion may not be susceptible to this same kind of speed subtraction (Durgin, Reed, \& Tigue, 2007), and that perceived biomechanical speed of self-motion is related to stride frequency (Durgin et al., 2007), are findings that were facilitated by being able to control the interaction between perception and action in ways that VR makes easier to achieve (although we did also develop a specialized robotic treadmill for the latter work). Thus, although biases in the perception of distance in VR have not been eliminated, it is possible to study variables such as perceived visual speed by modeling higher order relationships that are not disturbed by scalar errors in the perception of distance.

Graphics systems today are much faster than those of a decade ago, and their use has become more sophisticated. Still, most head-mounted displays (HMDs) have evident optical pincushion distortions (the optically correct Sensics HMDs are a notable exception). Although correcting for these distortions has not been a standard practice until recently, correction is now relatively easy to achieve in commercial software packages. The need for such correction is evident when observers are en- 
couraged to scan a scene. In an initial study of demand characteristics in the perception of slope (Russell \& Durgin, 2008; discussed in Durgin et al., 2009), we noticed that the perceived surface orientation of our virtual hills appeared to dynamically distort when we scanned up and down. Although a study using these uncorrected displays produced the anticipated differences in judgments of slope based on the demand characteristics of wearing a backpack (Russell \& Durgin, 2008), we have noted that many prior studies of slope perception in VR did not use optical correction. For example, Proffitt et al. (1995) reported what they regarded as "normative" data that include data from very primitive VR (e.g., uncorrected optics, refresh rates of about $10 \mathrm{~Hz}$, and lags of several hundred milliseconds). In retrospect, it seems likely that their participants depended on a pictorial cue (path edge convergence) that turned the slope estimation task into a cognitive problem-solving task. Other limitations of their design and measurement techniques have recently come to light (Bridgeman \& Hoover, 2008; Durgin, Hajnal, Li, Tonge, \& Stigliani, 2010).

As an alternative model of using VR to study slope perception, Li and Durgin (2009) studied the effect of the direction of gaze on downhill slope perception. They used both virtual hills and real (smaller) surfaces to collect slope judgments when observers were positioned at different horizontal distances from the surfaces so that their view of the target surface was either more parallel with the surface (as when standing back from the edge of the top of a hill) or more frontal to it (as when standing nearer the edge and looking more directly down onto the surface). Again, rather than directly interpreting the verbal judgments, Li and Durgin modeled the relationship between optical slant (the orientation of the surface relative to the line of gaze), gaze orientation, and perceived slope. Using a simple geometric model, they found that the functions relating true/simulated optical slant to (inferred) perceived optical slant became appropriately aligned when the model assumed that the perceived direction of gaze was misperceived by a constant gain factor. The analysis, again, depends entirely on comparing differences in representations of perceived optical slant without assuming that the scaling in VR corresponds to that in the real world. The overestimation of perceived head and gaze orientation was then measured separately in two further experiments and found to fit the predictions from the VR data.

The model developed by Li and Durgin (2009) showed that the perception of optical slant was exaggerated in both VR and the real world, but that the perception of head orientation was also (and similarly) exaggerated. As a consequence, for horizontal surfaces, the two biases should cancel each other out, but for uphill, sloped surfaces, the two errors would tend to combine to exaggerate the perceived slope, thus providing a new account of errors in uphill slope perception. For downhill surfaces, the two biases partly compensate for each other, resulting in a shallower perception of downhill slopes when standing at the edge than when standing farther back. Indeed, whereas the mathematical function relating optical slant to perceived optical slant was different in VR than in the real world (a scaling difference, which was not surprising, given the myriad differences in visual information available in the two cases), the functions relating perceived head and gaze orientation to perceived slope generalized across both contexts. What is particularly important about this example is that, rather than attempting to reproduce a known pattern of data in $\mathrm{VR}, \mathrm{Li}$ and Durgin discovered the geometric relationship using VR and then confirmed that it extended to the real world, using small-scale wooden surfaces.

It is important to note that $\mathrm{Li}$ and Durgin (2009) used a software shader in Virtools, a commercial rendering system, to correct for the pincushion distortion produced by the optics of their nVis HMD. Pincushion distortion results from imperfectly corrected optics in the HMD, and its effects are illustrated in Figure 1. Kuhl, Thompson, and Creem-Regehr (2009) recently described their technique for correcting and calibrating their nVis HMD, and they arrived at values very similar to ours $\left(42^{\circ} \times 34^{\circ}\right)$ for their true display size, although our optics appear to have produced greater pincushioning than theirs prior to correction. We recommend consulting their strategy for correcting and calibrating an HMD.

Kuhl et al. (2009) reported that the presence or absence of pincushion correction (at the levels they tested) had no effect on judgments of distance. However, our informal observations regarding the perception of slanted surfaces with and without correction suggest that there might be
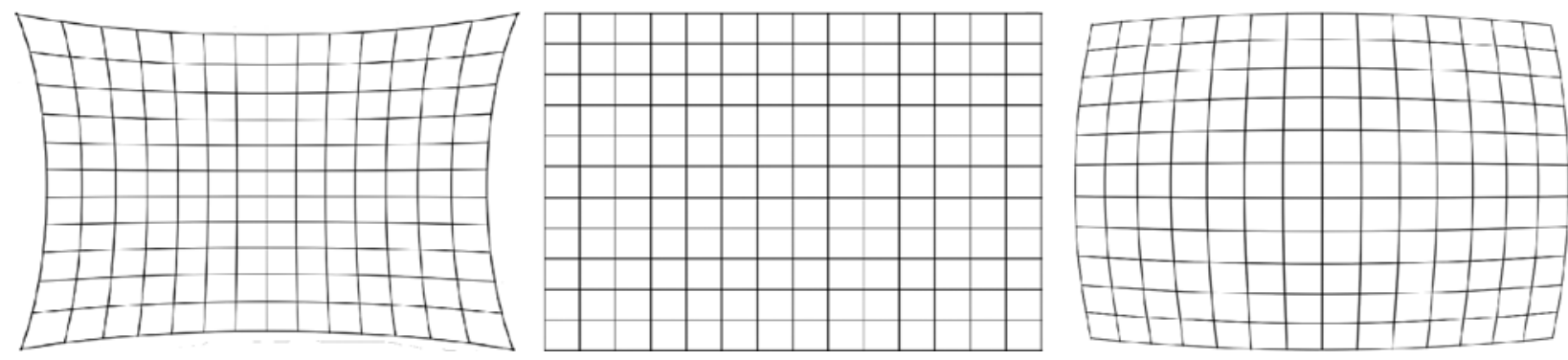

Figure 1. Pincushion distortion (left) means that a rectangular image (middle) will end up being displayed to the eye appearing compressed (left). Most of the geometrical distortion is in the periphery, although the center of the display is also minified. Pincushion correction algorithms spherically predistort the image in the opposite direction (right) so that the optical pincushion distortion ends up producing the originally intended rectangular image. 
measurable effects on slope perception. Moreover, rather than approaching the problem in the classic way of looking for absolute differences in slope estimates, we again sought to detect higher order differences in perception that might emerge in log space (exponents of power functions).

Most VR displays use stereoscopic disparity as a source of information about distance. Pincushion distortion would cause disparity information in the outer corners of the display to be exaggerated relative to that in the middle of the display. In theory, this could tend to make various parts of the lower and upper parts of the display appear nearer and farther than appropriate and thus produce evidence that the slope is both shallower and steeper than intended. Similar effects could arise from the 2-D distortion of texture gradients in the image. The expected outcome should be an increase in uncertainty and a concomitant reduction in sensitivity. This could be reflected as a decrease in the exponent of the power function-a compression of the perceptual scaling of slant perception.

Moreover, because there is a great deal of variability in the manner with which people may provide numeric estimates of slope, the advantage of summarizing estimates in terms of power function exponents is that the exponent of the power function can retain information about the rate with which slope information (for example) is used, while ignoring the absolute scale (which might otherwise add a great deal of between-subjects variance).

We therefore expected that the effect of pincushion distortion would be to reduce the exponent of a power function fit to the data by muting sensitivity to differences between slopes. To confirm our subjective impressions that pincushion correction caused perceptual instability during head motions, we also collected ratings of stability. We expected that direct ratings of the stability of scene geometry during head motions would be higher for a corrected display than for an uncorrected display.

\section{METHOD}

\section{Participants}

Twenty-four Swarthmore College undergraduate students who were naive to the hypotheses were reimbursed $\$ 5$ for their participation.

\section{Apparatus}

Displays were rendered to an nVis HMD, with nominal XVGA resolution updated at $60 \mathrm{~Hz}$ using Virtools 4.1 rendering software. Head orientation was monitored by a Vicon optical tracking system with minimal lag. A participant sat in a comfortable chair while looking at displays that were either corrected using an HLSL shader or presented uncorrected. Our correction constant was .3 (see Kuhl et al., 2009, for an explanation of this parameter).

\section{Virtual Environment}

The virtual environment was limited to a single rough surface that was composed of a flat sand-like texture and a plethora of jagged 3-D "rocks" that protruded slightly from the surface at irregular intervals. In addition, a small white ball on the surface of the hill served as a fixation point. The ball was $2 \mathrm{~m}$ from the observer (either directly ahead at eye level or along a line that was declined $30^{\circ}$ from horizontal). For slope estimation, participants were required to fixate the ball and were discouraged from moving their heads. For "distortion" judgments, they were required to move their heads vertically between the straight-ahead position and the ball in its lower location. Note that in the forward-gaze condition, the blue sky was visible beyond the end of the surface for the lowest three orientations. In the downward-gaze condition, the edge of the surface was not visible. Each view of the $12^{\circ}$ surface is shown in Figure 2.

\section{Design and Procedure}

The experiment had two parts (slope estimation and stability judgments). The slope estimates were always completed first, in two blocks. In one block, gaze was forward. In the other block, gaze was declined. The order of these blocks alternated between subjects. In each block of trials, five different slopes were tested in each optical condition (10 trials total per block). The simulated slopes were $6^{\circ}, 12^{\circ}, 18^{\circ}, 24^{\circ}$, and $30^{\circ}$ relative to the horizontal slope. The order of presentation was randomized within each block. A black screen appeared for $1 \mathrm{sec}$ between trials so that changes in correction and surface orientation would be masked. Slope judgments were made verbally in degrees, ranging from 0 for horizontal to 90 for vertical. Following the initial two blocks of verbal slope estimates, the second part of the experiment presented three slopes $\left(6^{\circ}, 18^{\circ}\right.$, and $30^{\circ}$ ) in each of the two optic conditions (6 trials in random order). Participants rated the amount of distortion experienced during head movement on a scale of 1 (completely stable) to 10 (very distorted). It was explained to participants that, as they moved their heads on each trial, they should try to notice whether the slanted surface appeared to rotate and/or distort, or whether it appeared fully rigid and stable.

\section{Analysis}

Angle estimates and slopes were converted to logarithms (after adding 1 , to eliminate zeros), and the slopes of least-squares fits

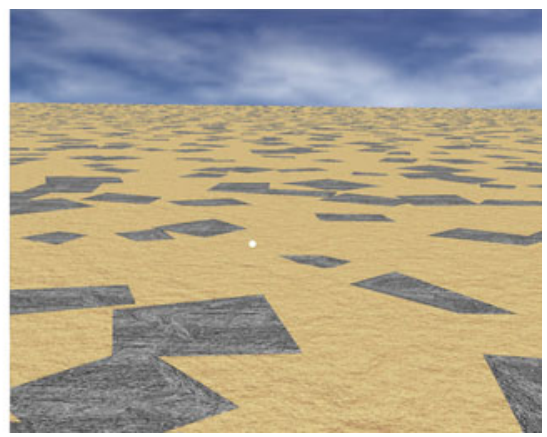

Figure 2. Sample frames from the experimental displays. Each represents a $12^{\circ}$ slope. The one on the left is depicted with gaze declined by $30^{\circ}$ from horizontal. The image on the right was viewed with horizontal forward gaze toward the white ball. 
(i.e., power function exponents) were computed for each participant for each head orientation and optical correction state. Note that the use of the slope in $\log -\log$ space to provide a scale-free summary of a set of data does not require that the data have the form of a power function. Rather, this use of a power function exponent is a one-parameter model that captures spread while neglecting scale. Scale is contained in the second parameter of a power functionthe intercept in $\log -\log$ space, which we ignore in this case. Rawrating scale measures were compared according to optical correction condition.

\section{RESULTS}

\section{Slope Estimates}

We predicted greater sensitivity (higher exponents) to slope differences when optical correction was applied. A 2 (gaze: up, down) $\times 2$ (optical correction: corrected, uncorrected) repeated measures ANOVA revealed only a marginally reliable effect of optical correction $[F(1,69)=$ $2.70, p=.0528$, one-tailed] in the predicted direction. However, the analysis revealed a highly reliable effect of gaze $[F(1,69)=26.4, p<.0001]$, likely indicating a strong influence of the presence of a horizon line formed by the top of the hill in the gaze-up condition (see Figure 2), in which the average exponents (1.13) were much higher than those in the gaze-down condition (0.52). Because our predictions regarding pincushion correction were made on the basis of expected distortions of optical slant information (from texture and stereopsis), whereas the horizon line could have acted as a cognitive reference, the apparently large effect of the hilltop horizon line was treated as a confounding influence, and data from the gaze-down condition, in which the horizon was never visible, were analyzed separately.

The median estimation data are shown in Figure 3, in $\log -\log$ space, as a function of optical correction for the gaze-down condition. When a repeated measures ANOVA was conducted on the gaze-down data separately, the average exponents in the uncorrected pincushion condition $(0.37, S E=0.17)$ were found to be reliably lower than those in the optically corrected condition $(0.68, S E=0.21)$ $[F(1,23)=8.06, p=.0093]$. Thus, as expected, pincushion

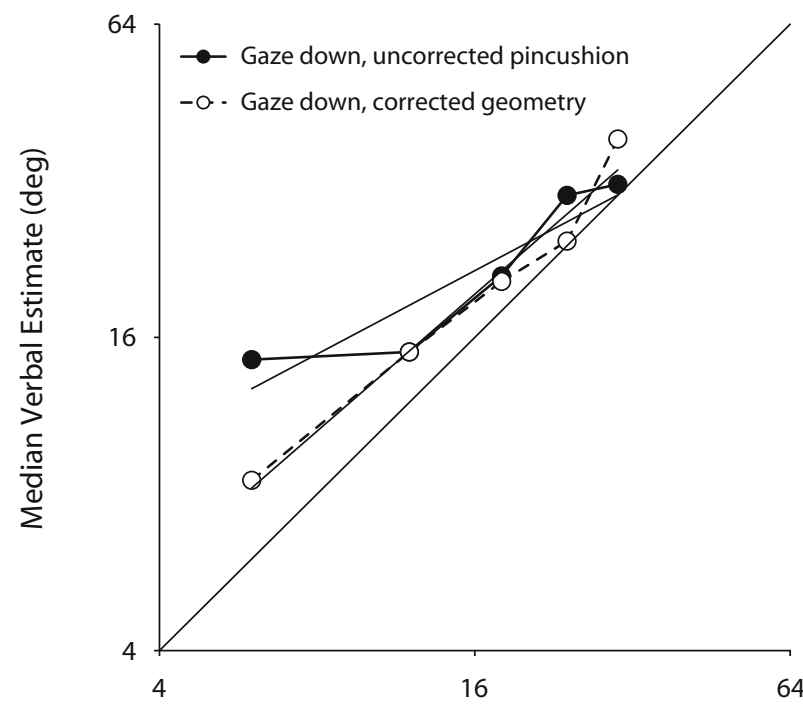

Simulated Surface Orientation (deg)

Figure 3. Median estimates of geographical slant with and without pincushion correction are shown for the gaze-down condition in log-log space. These medians suggest that in the presence of pincushion distortion, surface orientations were less well differentiated from each other. Power function exponents represent the best-fitting slope in $\log -\log$ space. Statistics based on analyses of power function exponents revealed that this pattern of compressed responding was statistically reliable.

distortion seems to have degraded the scaling of the optical slant information required to estimate optical slant in the gaze-down condition. Note that, because it is sensitive to arbitrary scale selection, an analysis of least-squares fits in linear space did not reliably detect even the large difference in the gaze-down condition $[t(23)=1.47, p=.155]$ indicated by the analysis in log-log space. Our argument is not that there is no effect in linear space, but that such analyses are likely to be less sensitive to theoretically important differences because they are scale sensitive, even when the theoretical question under investigation is not.

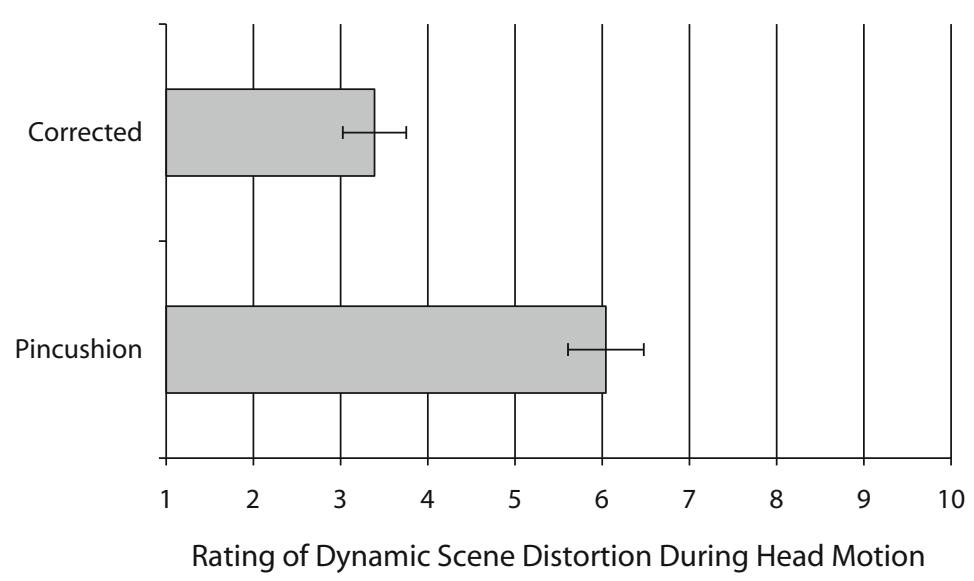

Figure 4. Ratings of dynamic scene distortion during head motion as a function of whether pincushion distortion is present or corrected. Standard errors of the means are shown. 


\section{Ratings of Dynamic Distortion}

Pincushion distortion is not obvious during static viewing of irregular textures, but it produces dynamic distortions of geometry when the head is moved. Average ratings of dynamic distortion are shown in Figure 4. For our corrected displays, the average rating of dynamic distortion was only 3.4 (on a 1 to 10 scale), whereas the average rating in the pincushion condition was 6.0 , which was reliably greater $[t(23)=4.45, p=.0002]$.

\section{Summary of Results}

Uncorrected pincushion distortion in our $\mathrm{nVis}$ HMD had measureable consequences for the extraction of spatial parameters, such as surface orientation. Pincushion distortion also produced noticeable dynamic distortion when the head was reoriented. Note that in our study of perceived surface orientation, we did not allow head movements because we sought to isolate static effects of pincushion distortion. It is likely that dynamic aspects of pincushion distortion could add additional noise to the perception of surface layout. Although human perception is highly adaptive, studies that intend to investigate active perceptual exploration of spatial parameters using HMDs for immersive VR should seek to correct their optics.

\section{DISCUSSION}

VR is a powerful tool for studying the interaction between perception and action. Whereas VR systems remain imperfect in their scaling of space, we have recommended using scale-free strategies for harnessing the power of VR.

One strategy, the use of power function exponents, is both a theoretical strategy and a methodological one: Whereas the variability in our participants' slope judgments meant that standard analyses of means would be unable to detect effects of pincushion distortion on slope perception, the use of scale-free, one-parameter representations of data (such as power function exponents) have, once again, provided a way of extracting the essential pattern of data we expected: When slope estimates must be based on optical slant, pincushion distortion adds uncertainty to estimates. This can be measured as a proportionally compressed set of estimates of slope (a lower exponent) for the distorted case as opposed to the corrected case, as we have shown. Verbal judgments are prone to biases, but when considered in aggregate and transformed into higher order (scale-free) statistics, they seem to be quite sensitive to subtle perceptual effects. Messing and Durgin (2005) depended on the theoretical value of higher order statistics to detect the effect of a shifted horizon. In the present study, we have emphasized that scale-free analyses are better suited to detect effects of pincushion distortion on the static perception of spatial variables, such as surface orientation.

Our second scale-free strategy was to take advantage of perceptuomotor interaction, but to do so in a way that demands as few assumptions about the stimulus presented as possible. When Li and Durgin (2009) discovered that the proprioception of head orientation seemed to play a role in the misperception of downhill slope, they investigated this by creating contexts in which the interaction of the observer with the virtual world included head motions. (They did not, for example, as we did in the present study, allow participants to keep their heads declined for a block of trials.) Their modeling, however, could remain neutral in its assumptions about the verisimilitude of VR because it did not depend on the form of the relationship between perceived and simulated optical slant; it only assumed that there was a stable relationship. That is, it did not depend on the scale's being accurate. Nonetheless, because metric space perception itself was under investigation (i.e., slope perception) and head movements were required, the elimination of dynamic distortions due to pincushion distortion seems important to their use of immersive VR.

Whereas immersive VR remains an imperfect medium for the study of perception, both the use of scale-invariant metrics (such as power function exponents) and the carefully controlled engagement of perception and action as a principled research strategy hold great promise while we wait for future improvements.

\section{AUTHOR NOTE}

The present research was supported by a Swarthmore College faculty research grant. Correspondence concerning this article should be addressed to F. H. Durgin, Department of Psychology, Swarthmore College, 500 College Avenue, Swarthmore, PA 19081 (fdurgin1@ swarthmore.edu).

\section{REFERENCES}

AKAGI, M., \& DURGIN, F. (2007). Accurate perception of visual space from live-video in a head-mounted display [Abstract]. Journal of Vision, 7, 283a. doi:10.1167/7.9.283

Bridgeman, B., \& Hoover, M. (2008). Processing spatial layout by perception and sensorimotor interaction. Quarterly Journal of Experimental Psychology, 61, 851-859.

Creem-Regehr, S. H., Gooch, A. A., Sahm, C. S., \& Thompson, W. B. (2004). Perceiving virtual geographical slant: Action influences perception. Journal of Experimental Psychology: Human Perception \& Performance, 30, 811-821. doi:10.1037/0096-1523.30.5.811

Durgin, F. H., Baird, J. A., Greenburg, M., Russell, R., ShaughNessy, K., \& Waymouth, S. (2009). Who is being deceived? The experimental demands of wearing a backpack. Psychonomic Bulletin \& Review, 16, 964-969. doi:10.3758/PBR.16.5.964

Durgin, F. H., \& Gigone, K. (2007). Enhanced optic flow speed discrimination while walking: Contextual tuning of visual coding. Perception, 36, 1465-1475.

Durgin, F. H., Gigone, K., \& Scott, R. (2005). The perception of visual speed while moving. Journal of Experimental Psychology: Human Perception \& Performance, 31, 339-353.

Durgin, F. H., Hajnal, A., Li, Z., Tonge, N., \& Stigliani, A. (2010). Palm boards are not action measures: An alternative to the twosystems theory of geographical slant perception. Acta Psychologica, 134, 182-197. doi:10.1016/j.actpsy.2010.01.009

Durgin, F. H., Reed, C., \& Tigue, C. (2007). Step frequency and perceived self-motion. ACM Transactions in Applied Perception, 4, 1-27.

Grodner, D., Pietsch, C., \& Durgin, F. H. (2009). The use of categorical features in adult spatial reorientation. In N. Taatgen \& H. van Rijn (Eds.), Proceedings of the 2009 Meeting of the Cognitive Science Society (pp. 280-285). Amsterdam: Cognitive Science Society.

Harris, L. R., Morgan, M. J., \& Still, A. W. (1981). Moving and the motion after-effect. Nature, 293, 139-141.

Interrante, V., Anderson, L., \& Ries, B. (2006). Distance perception in immersive virtual environments, revisited. In Proceedings of the IEEE Conference on Virtual Reality (pp. 3-10). Washington, DC: IEEE Computer Society. 
Interrante, V., Ries, B., Lindquist, J., \& Anderson, L. (2007). Elucidating the factors that can facilitate veridical spatial perception in immersive virtual environments. In Proceedings of the IEEE Conference on Virtual Reality (pp. 11-18). Washington, DC: IEEE Computer Society.

Kuhl, S. A., Thompson, W. B., \& Creem-Regehr, S. H. (2009). HMD calibration and its effects on distance judgments. ACM Transactions on Applied Perception, 6, 19.

Li, Z., \& Durgin, F. H. (2009). Downhill slopes look shallower from the edge. Journal of Vision, 9, 1-15. doi:10.1167/9.11.6

Loomis, J. M., Blascovich, J. J., \& BeAll, A. C. (1999). Immersive virtual environment technology as a basic research tool in psychology. Behavior Research Methods, Instruments, \& Computers, 31, 557-564.

Loomis, J. M., \& KNAPP, J. M. (2003). Visual perception of egocentric distance in real and virtual environments. In L. J. Hettinger \& M. W. Haas (Eds.), Virtual and adaptive environments (pp. 21-45). Mahwah, NJ: Erlbaum.

Messing, R., \& Durgin, F. H. (2005). Distance perception and the visual horizon in head-mounted displays. ACM Transactions on Applied Perception, 2, 234-250. doi:10.1145/1077399.1077403

Mohler, B. J., Thompson, W. B., Creem-Regehr, S. H., WillemSEN, P., Pick, H. L., \& Rieser, J. J. (2007). Calibration of locomotion resulting from visual motion in a treadmill-based virtual environment. ACM Transactions on Applied Perception, 4, 1-15. doi: $10.1145 / 1227134.1227138$

OoI, T. L.,Wu, B., \& HE, Z. (2001). Distance determined by the angular declination below the horizon. Nature, 414, 197-200.
Powers, W. T. (1973). Behavior: The control of perception. Chicago: Aldine.

Proffitt, D. R., Bhalla, M., Gossweiler, R., \& Midgett, J. (1995). Perceiving geographical slant. Psychonomic Bulletin \& Review, 2, 409-428.

Russell, R., \& Durgin, F. H. (2008). Demand characteristics, not effort: The role of backpacks in judging distance [Abstract]. Journal of Vision, 8(6), 755a.

Teghtsoonian, R., \& Teghtsoonian, M. (1970). Scaling apparent distance in a natural outdoor setting. Psychonomic Science, 21, 215-216.

Thompson, W. B., Willemson, P., Gooch, A. A., Creem-Regehr, S. H., Loomis, J. M., \& BeAll, A. C. (2004). Does the quality of the computer graphics matter when judging distance in visually immersive environments? Presence: Teleoperators \& Virtual Environments, 13, 560-571.

Wallach, H., \& Flaherty, E. W. (1975). A compensation for field expansion caused by moving forward. Perception \& Psychophysics, 17, 445-449.

Warren, W. H., Kay, B. A., Zosh, W. D., Duchon, A. P., \& Sahuc, S (2001). Optic flow is used to control human walking. Nature Neuroscience, 4, 213-216.

Wolpert, D. M., \& Flanagan, J. R. (2001). Motor prediction. Current Biology, 11, R729-R732.

(Manuscript received November 12, 2009; revision accepted for publication February 16, 2010.) 